\title{
BIBLIOGRAPHY
}

1. B. Germansky, An identity in the theory of the generalized polynomials of Jacobi, Proc. Amer. Math. Soc. 9 (1958), 953-956.

2. G. Szegö, Orthogonal polynomials, New York, 1959.

3. B. Germansky, On the Fekete systems of an arc of a circle, Riveon Lematematika 3 (1949), 6 (in hebraisch, mit einer Zusammenfassung in englisch).

4. Karl Heun, $Z$ ur Theorie der Riemannschen Funktionen zweiter Ordnung mit vier Verzweigungspunkten, Math. Ann. 33 (1889), 165, Gleichung (10).

Mathematical Institute,

TeChNical UNiversity OF BerLin

\section{ON REDUNDANCY OF POWERS AND THE MOMENT PROBLEM}

DAVID S. GREENSTEIN

1. Introduction. Let $\psi(t)$ be a bounded nondecreasing function with infinitely many points of increase such that the mass distribution $d \psi(t)$ has moments of all orders. Under these conditions, the powers $t^{n}(n=0,1, \cdots)$ are linearly independent elements of $L^{2} \psi$, which consists of all $\psi$-measurable $f(t)$ for which

$$
\|f(t)\|_{\psi}^{2}=\int_{-\infty}^{\infty}|f(t)|^{2} d \psi(t)<\infty .
$$

We shall call any of the above powers redundant if it belongs to the closed linear manifold of $L_{\psi}^{2}$ spanned by all higher powers. The author has shown [2, Theorem 2.4] that, for absolutely continuous $d \psi(t)$, if the powers span $L^{2} \psi$, then each power is redundant. This result, while weaker than the Müntz-Szasz Theorem, does not require that $d \psi(t)$ have compact support.

In the present paper, redundancy is studied for general $d \psi(t)$, thus placing the above result in a more natural context. Our investigation, using the theory of the Hamburger moment problem, allows us to study some properties of extremal solutions of the moment problem and to add to Wright's work [6] on backward extension of moment sequences.

2. Some needed results. The following theorem of M. Riesz $\left[4 ; 5\right.$, p. 60] is important for polynomial approximation in $L_{\psi}^{2}$ :

Received by the editors March 6, 1961. 
TheOREM 2.1. The powers $t^{n}(n=0,1, \cdots)$ span $L_{\psi}^{2}$ if and only if one of the following holds:

(1) The moments of $d \psi(t)$ determine it uniquely.

(2) The mass distribution $d \psi(t)$ is one of $R$. Nevanlinna's extremal solutions of an undetermined Hamburger moment problem.

For a complete discussion of extremal solutions the reader is referred to $[3 ; 5$, p. 60$]$. The properties which we need are given by the following.

THEOREм 2.2. Given an undetermined Hamburger moment sequence, there exists a continuum of mass distributions called extremal solutions having the following properties:

(1) The support of each extremal solution is a denumerable set consisting of the zeros of an entire function of minimal exponential type, the mass concentrated at each of these points being greater than that concentrated by any other mass distribution with the same moments.

(2) For every real $t_{0}$ there exists one (and hence only one) extremal solution having $t_{0}$ in its support and infinitely many extremal solutions having no mass concentrated at $t_{0}$.

It should be mentioned that associated with every Hamburger moment sequence $\left\{\mu_{n}\right\}$ there is a maximal mass function $\rho(x)$ whose value at each real $x$ gives the maximum mass concentrated at $t=x$ by any $d \psi(t)$ having the $\left\{\mu_{n}\right\}$ as moments. (An explicit formula for computing $\rho(x)$ from the orthonormal polynomials associated with $\left\{\mu_{n}\right\}$ is given in $\left[5\right.$, p. 44].) If we let $\rho^{(2)}(x)$ be the maximal mass function associated with $\left\{\mu_{n+2}\right\}$, then there is the following result due originally to Hamburger [5, p. 72]:

THEOREM 2.3. A necessary and sufficient condition for the Hamburger moment sequence $\left\{\mu_{n}\right\}$ to be determined is that at least one of the two quantities $\rho(0), \rho^{(2)}(0)$ is equal to zero.

3. Theorems on redundancy. The following theorem, a proof of which can be found in [2], is our basic tool for studying redundancy:

Theorem 3.1. If $P(t)$ denotes any polynomial with $P(0)=0$, then the infimum of $\|1-P(t)\|_{\psi}^{2}$, taken over all $P(t)$, is equal to $\rho(0)$ :

By combining Theorems 2.1 and 3.1, we have the following result.

THEOREM 3.2. The necessary and sufficient conditions for redundancy of 1 in $L^{2} \psi$ are that $d \psi(t)$ be uniquely determined by its moments and have no mass concentrated at $t=0$. If 1 is redundant then the powers $t^{n}(n=1,2, \cdots) \operatorname{span} L^{2} \psi$. 
To investigate the redundancy of $t^{k}$ for positive $k$, consider the mass distribution $d \phi(t)=t^{2 k} d \psi(t)$. For any polynomial $P(t),\|P(t)\|_{\phi}$ $=\left\|t^{k} P(t)\right\|_{\psi}$. Hence $t^{k}$ is redundant in $L^{2} \psi$ if and only if 1 is redundant in $L_{\phi}^{2}$. This fact, combined with Theorem 2.3, yields the following:

THEOREM 3.3. The mass distribution $d \psi(t)$ is uniquely determined by its moments if and only if 1 or $t$ is redundant in $L^{2} \psi$. If $t$ is redundant then 1 will be redundant if and only if $d \psi(t)$ has no mass concentrated at $t=0$.

Now suppose that $k>1$ and that $t^{k}$ is redundant in $L^{2} \psi$. Then $d \phi(t)$ is uniquely determined by its moments, from which it also follows that each of the mass distributions $t^{2 n} d \psi(t)(n=0, \cdots, k-1)$ is also uniquely determined by its moments. Since for $n=1, \cdots$, $k-1, t^{2 n} d \psi(t)$ has no mass concentrated at $t=0$, it follows that $t^{n}(n=1, \cdots, k-1)$ are also redundant in $L_{\psi}^{2}$. Hence the following theorem is true.

THEOREM 3.4. If $t^{k}$ is redundant in $L_{\psi}^{2}$ for some integral $k>1$, then all lower positive integral powers are also redundant in $L \psi$, which is spanned by the powers.

Now let us see whether redundancy of higher powers is implied by redundancy of a given power. Suppose that for some positive integer $k, t^{k-1}$ is redundant in $L^{2} \psi$ but $t^{k}$ is not. Then it follows that 1 is the only redundant power in the $L^{2}$ space determined by $t^{2(k-1)} d \psi(t)$. But this space is isometrically mapped onto $L_{\phi}^{2}$ by the correspondence $f(t) \leftrightarrow t^{-1} f(t)$. (For $k=1$, note that $d \psi(t)$ cannot have mass concentrated at $t=0$.) Hence $L_{\phi}^{2}$ is spanned by all the powers without anv redundancy. By Theorems 3.3 and 2.1 , it follows that $d \phi(t)$ is a Nevanlinna distribution; i.e., an extremal solution of an undetermined Hamburger problem.

By a reversal of the reasoning just used, it is easily shown that for a Nevanlinna distribution $d \phi(t)$ with no mass concentrated at $t=0$, $d \psi(t)=t^{-2 k} d \phi(t)$ is such that $t^{k-1}$, but not $t^{k}$ is redundant in $L^{2} \psi$. For $k>1$ this will still be true if we add mass at the origin to $d \psi(t)$. Thus the following has been proven.

THEOREM 3.5. For each positive integer $k$, there exist mass distributions $d \psi(t)$ for which $t^{k-1}$ is redundant in $L^{2} \psi$ but $t^{k}$ is not. The necessary and sufficient condition that $d \psi(t)$ exhibit such behavior is that $t^{2 k} d \psi(t)$ be a Nevanlinna distribution, with the additional restriction that if $k=1$, $d \psi(t)$ have no mqss concentrated at $t=0$.

Theorem 3.5 rules out the possibility of any analogue of the Müntz- 
Szasz Theorem for general $L^{2} \psi$. However, if the support of $d \psi(t)$ cannot be the support of a Nevanlinna distribution, then once it is known that the powers $\operatorname{span} L^{2} \psi$, they must all be redundant, with the possible exception of 1 . An example of such a condition on the support is that it have a limit point, as would be the case for absolutely continuous $d \psi(t)$ (thus proving the author's earlier result in [2]). Another condition can be derived from Theorem 2.2 and a theorem on entire functions [1, Theorem 2.10.3]; viz., that the support of $d \psi(t)$ include the real numbers $t_{n}$, where $\left|t_{1}\right|<\left|t_{2}\right|<\cdots$ and $\sum t_{n}^{-1}$ diverges.

4. Some properties of extremal solutions. Let $d \phi(t)$ be a Nevanlinna distribution having no mass concentrated at $t=0$. As was seen in the proof of Theorem 3.5, the mass distribution $t^{-2} d \phi(t)$ is uniquely determined by its moments. If, however, we take $d \psi(t)=t^{-2} d \phi(t)$ $+d \alpha(t)$, where $d \alpha(t)$ has all of its mass concentrated at $t=0$, then neither 1 nor $t$ will be redundant in $L^{2} \psi$. Hence by Theorem 3.3, $d \psi(t)$ is not uniquely determined by its moments. However, $d \psi(t)$ must be a Nevanlinna distribution.

To show this, note that if $f(t) \in L^{2} \psi$, then

$$
\|f(t)-f(0)\|_{\psi}^{2}=\int_{-\infty}^{\infty}|f(t)-f(0)|^{2} t^{-2} d \phi(t) .
$$

Thus $f(t)-f(0)$ is in the $L^{2} \psi$ span of $t, t^{2}, \cdots$, from which it follows that the powers $t^{n}(n=0,1, \cdots)$ span $L^{2} \psi$. By Theorem 2.1, $d \psi(t)$ must be a Nevanlinna distribution. That, conversely, removing the mass at $t=0$ from a Nevanlinna distribution $d \psi(t)$ having $t=0$ in its support yields a mass distribution uniquely determined by its moments is already known (see [5, p. 45]). By Theorem 3.5, it follows that $t^{2} d \psi(t)$ is a Nevanlinna distribution.

By translating $d \psi(t)$, it is easily seen that the following theorem is valid:

Theorem 4.1. Let $d \psi(t)$ be a Nevanlinna distribution. If $t_{0}$ is in the support of $d \psi(t)$, then $\left(t-t_{0}\right)^{2} d \psi(t)$ is also a Nevanlinna distribution. If $t_{1}$ is not in the support of $d \psi(t)$, then $\left(t-t_{1}\right)^{-2} d \psi(t)$ is determined by its moments and becomes a Nevanlinna distribution when mass is added at $t_{1}$.

As an immediate corollary of Theorem 4.1, we have the following:

Theorem 4.2. Let $S$ be the support of a Nevanlinna distribution. Then any set differing from $S$ by a set of finite cardinality is also the support of a Nevanlinna distribution. 
Perhaps Theorem 4.2 will be of use in determining precisely what sets can be the support of a Nevanlinna distribution.

5. Backward extension of moment sequences. Let $\mu_{0}, \mu_{1}, \cdots$ be the moments of some mass distribution on the real line. Any Hamburger moment sequence $\lambda_{0}, \lambda_{1}, \cdots$ such that $\lambda_{n}=\mu_{n-2}(n=2,3, \cdots)$ is called a backward extension of $\left\{\mu_{n}\right\}$. For convenience, we write $\mu_{-1}$ for $\lambda_{1}$ and $\mu_{-2}$ for $\lambda_{0}$, allowing $\left\{\mu_{n}\right\}$ to represent the original unextended moments.

In [6] Wright showed that if $\left\{\mu_{n}\right\}$ is undetermined, backward extensions are possible, the region of all possible ordered pairs $\left(\mu_{-2}, \mu_{-1}\right)$ being closed, convex, and bounded by a parabola. He showed that only the boundary points of the region correspond to determined backward extensions.

We shall relate the extreme values of $\left(\mu_{-2}, \mu_{-1}\right)$ to Nevanlinna distributions and give an approximation-theoretic computation of Wright's parabola. First of all, every Nevanlinna distribution $d \phi(t)$ having $\left\{\mu_{n}\right\}$ as its moments and having no mass concentrated at $t=0$ gives backward extensions by means of the mass distributions $t^{-2} d \phi(t)+c d \alpha(t)$, where $c \geqq 0$ and $d \alpha(t)$ has all of its mass concentrated at $t=0$. By Theorem 4.1, these are Nevanlinna distributions for $c>0$ and distributions with determined moments for $c=0$.

The analytic representation for the Stieltjes transforms of the above $d \phi(t)$ [5, Theorem 2.13] shows that these mass distributions, each having different $\mu_{-1}$, yield every real number as a possible value of $\mu_{-1}$. By Theorem 3.5, all determined backward extensions come from the mass distributions $t^{-2} d \phi(t)$. Hence the points on Wright's parabola must all come from these mass distributions, as will be independently verified by our computation.

To compute all possible $\mu_{-2}$ corresponding to fixed $\mu_{-1}$, let $d \psi(t)$ be any mass distribution such that

$$
\int_{-\infty}^{\infty} t^{n} d \psi(t)=\mu_{n} \quad(n=-1,0,1, \cdots),
$$

and such that the above integral exists for $n=-2$. Let $\omega_{n}(t)$ $(n=0,1, \cdots)$ be the orthonormal polynomials of $L^{2} \psi$.

Since $t^{-1}$ is an element of $L^{2} \psi$, let us compute the coefficients of its Fourier expansion $\sum C_{n} \omega_{n}(t)$. It is easily verified that $C_{n}=\mu_{-1} \omega_{n}(0)$ $+\chi_{n}(0)$, where $\chi_{n}(z)$ is the polynomial

$$
\chi_{n}(z)=\int_{-\infty}^{\infty}\left(\omega_{n}(z)-\omega_{n}(t)\right)(z-t)^{-1} d \psi(t) .
$$


Hence by Bessel's inequality,

$$
\int_{-\infty}^{\infty} t^{-2} d \psi(t) \geqq \sum_{n=0}^{\infty}\left(\mu_{-1} \omega_{n}(0)+\chi_{n}(0)\right)^{2}
$$

with equality if and only if 1 is redundant in the $L^{2}$ space determined by $t^{-2} d \psi(t)$, therefore if and only if $d \psi(t)$ is a Nevanlinna distribution. Furthermore, if $t^{2} d \beta(t)=d \psi(t)$, we have

$$
\mu_{-2}=\int_{-\infty}^{\infty} d \beta(t) \geqq \int_{-\infty}^{\infty} t^{-2} d \psi(t),
$$

with equality if and only if $d \beta(t)$ has no mass concentrated at $t=0$.

Finally, by combining (5.1) and (5.2), we have

$$
\mu_{-2} \geqq \sum_{n=0}^{\infty}\left(\mu_{-1} \omega_{n}(0)+\chi_{n}(0)\right)^{2}
$$

with equality if and only if $d \psi(t)$ is a Nevanlinna distribution and $\mu_{-2}=\int_{-\infty}^{\infty} t^{-2} d \psi(t)$. The inequality (5.3) gives Wright's parabola and verifies our previous assertion that points on the parabola come only from Nevanlinna distributions.

\section{REFERENCES}

1. R. P. Boas, Jr., Entire functions, Academic Press, New York, 1954.

2. D. S. Greenstein, Derivative manifolds and Taylor series in the mean, Trans. Amer. Math. Soc. 90 (1959), 312-322.

3. R. Nevanlinna, Asymptotische Entwickelungen beschränkter Funktionen und das Stieltjessche Momentenproblem, Ann. Acad. Sci. Fenn. (A) 18 (1922).

4. M. Riesz, Sur le problème des moments et sur le theorème de Parseval correspondant, Acta Sci. Math. Szeged 1 (1922-23), 209-225.

5. J. A. Shohat and J. D. Tamarkin, The problem of moments, Math. Surveys No. 1, Amer. Math. Soc., Providence, R. I., 1943 and 1950.

6. F. M. Wright, On the backward extension of positive definite Hamburger moment sequences, Proc. Amer. Math. Soc. 7 (1956), 413-422.

NORTHWESTERN UNIVERSITY 